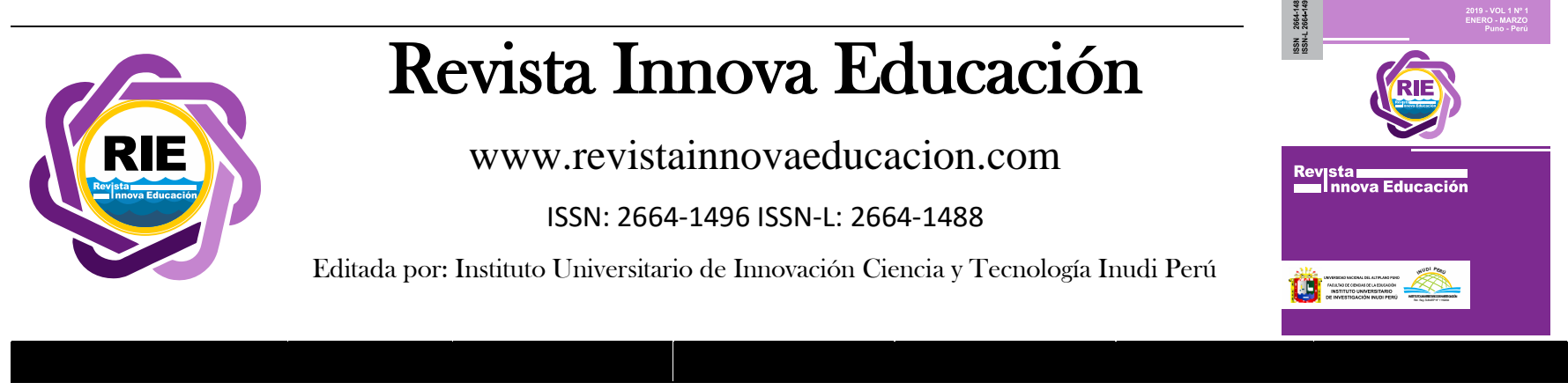

\title{
Migración rural-urbana de jóvenes y adolescentes en riesgo de exclusión social y educativa
}

\author{
Rural-urban migration of young people and adolescents at risk of social and educational \\ exclusion \\ Bethzabe Cotrado $^{1}$ (iD); Pedro Huayanca ${ }^{2}$ iD $;$ Haydee Nina \\ DOI: https://doi.org/10.35622/j.rie.2019.01.007
}

${ }^{1}$ Universidad Autónoma de Barcelona; email: bethzabe.cotrado@e-campus.uab.cat
${ }^{2}$ Universidad Nacional del Altiplano Puno, email: phuayanca@inudiperu.org

Recibido el 18/01/2019/ Aceptado el 27/01/2019

ARTíCULO ORIGINAL
PALABRAS CLAVE
educativa, exclusión
social, factores de
riesgo, jóvenes y
adolescentes,
migración rural-
urbana.

\section{KEYWORDS}

educational, social exclusion; risk factor's; rural-urban migration, youth and adolescents.

\begin{abstract}
El presente texto realiza una revisión de literatura científica con el objetivo de conocer las diferentes miradas e interpretaciones sobre migración rural - urbana de jóvenes y adolescentes en riesgos de exclusión social. La metodología aplicada en el marco del enfoque cualitativo permitió la organización, el estudio e interpretación de 25 investigaciones publicadas en la biblioteca digital de la Universidad Autónoma de Barcelona. Como resultado se llegó a comprender que la migración es un fenómeno complejo, dinámico y multifacético. En esta perspectiva emerge el tipo de movimiento migratorio rural-urbana que recae en el grupo de jóvenes y adolescentes que "salen en busca de trabajo" y los que "migran para estudiar". Esta población de jóvenes y adolescentes migrantes del campo a la ciudad es uno de los segmentos sumamente vulnerables. En esa condición, los sujetos jóvenes están expuestos a múltiples procesos dinámicos de exclusión social, educativa, laboral, política y cultural.
\end{abstract}

\footnotetext{
This article makes a review of scientific literature with the objective of knowing the different perspectives and interpretations on rural - urban migration of young people and adolescents at risk of social exclusion. The methodology applied in the framework of the qualitative approach allowed the organization, study and interpretation of 25 research published in the digital library of the Autonomous University of Barcelona. As a result, it was understood that migration is a complex, dynamic and multifaceted phenomenon. In this perspective emerges the type of rural-urban migration movement that falls on the group of young people and adolescents who "go out in search of work" and those who "migrate to study". This population of migrant youth and adolescents from the countryside to the city is one of the most vulnerable segments. In this condition, young subjects are exposed to multiple dynamic processes of social, educational, labor, political and cultural exclusion.
} 


\section{INTRODUCCIÓN}

El tema de la migración se ha convertido en uno de los fenómenos altamente complejo y dinámico de mayor relevancia a principios del siglo XXI (Tacoli \& Mabala, 2010). Desde Europa, Asia y pasando por América, nos enfrentamos a un proceso migratorio de alcance planetario de gran complejidad (Morales \& Rosario, 2012). Esta diversidad abarca procesos de transformación socioeconómica, demográfica, cultural y política (Tacoli \& Mabala, 2010), incluyendo cambios en la distribución desigual de recursos que, consecuentemente, repercute en la pérdida del estado de bienestar y en la exclusión de grandes sectores de la población (Ortega \& Ng, 2013). En medio de este proceso dinámico destaca la migración rural-urbana y los factores de riesgo de exclusión social y educativa de los jóvenes y adolescentes de áreas rurales. Los jóvenes y adolescentes de este ámbito son expuestos a altas tasas de vulnerabilidad, condiciones de extrema pobreza, marginación y discriminación. Son partícipes de escasas prestaciones laborales y reducido acceso a la educación, así como la pérdida de su identidad en el contexto urbano (Jurado y Tobasura, 2012). Bajo estas condiciones los sujetos jóvenes entran a ser parte de los problemas de riesgo de exclusión social como drogadicción, vandalismo, prostitución y violencia (Jurado y Tobasura, 2012). Por lo tanto, las investigaciones hacen evidente que la migración y exclusión social de esta población, es un fenómeno social que se intensifica en los últimos años con claras consecuencias. En señal de lo expuesto el artículo orientado desde la metodología cualitativa, presenta una revisión y análisis de las producciones científicas publicadas a partir del año 2010 al 2016. Concretamente se enfoca en las interpretaciones y perspectivas de la migración, exclusión, en los procesos de la migración rural-urbana y en los factores de riesgo de exclusión social y educativa.

\section{MÉTODO}

La metodología en el marco cualitativo inicia a partir de una amplia búsqueda, más no exhaustiva de la literatura científica sobre la migración rural-urbana de los jóvenes y adolescentes en riesgo de exclusión social y educativa. El mapeo se realiza en las bases de datos bibliográficos: Google Scholar y Trobador+ (portal de acceso a la biblioteca digital de la Universidad Autónoma de Barcelona). Para la selección de artículos se ha considerado tres criterios: la temporalidad, donde los documentos seleccionados se enmarcan cronológicamente entre 2010 y 2016. La adecuación, este segundo criterio establece que la investigación contiene el objetivo y se relaciona con el tema en concreto. Contenido de la información, tercer criterio cuya característica presenta rigor y calidad científica. Los criterios precedentes facilitaron identificar y seleccionar los artículos en base a la lectura del título, del resumen, y de las palabras clave.

La primera búsqueda se realiza en la base de datos de Google Scholar situando los descriptores “migración rural-urbana y exclusión social y adolescentes" y restringiendo el año entre 2010 a 2016, como resultado reporta $n=1490$ documentos. Esta cantidad desmedida de textos académicos recae en la decisión de utilizar el Trobador+. Esta base de datos digital especifica la búsqueda de documentos limitando el título, la fecha de publicación (últimos 5 años), el tipo de material (solo artículos) e idioma. Desde esa lógica, la búsqueda procede utilizando descriptores como: "migración rural - urbana" OR "Exclusión social" AND "jóvenes y adolescentes"; como resultado se visualiza $n=4$ artículos, se elige $n=2$. En el proceso siguiente de búsqueda se usa solamente "migración rural urbana" y reporta $n=39$ artículos, de los cuales se eligen $n$ $=16$ documentos. Por otro lado, se combina descriptores en inglés como: "rural-urban migration" AND "social exclusion" y se obtiene $\mathrm{n}=8$, se elige $\mathrm{n}=2$; "rural-urban migration" AND "Young people" $\mathrm{n}=92$, se escoge $n=6$ artículos; además en la búsqueda se combina con otras palabras claves como "migración campo-ciudad", "exclusión social" AND "migración", "urbanización" AND "factores de exclusión" y "Migración rural-urbana" AND "pobreza". Finalmente se identifican y define $\mathrm{n}=25$ artículos que cumplen los criterios de selección preestablecidos para su respectivo análisis.

\section{RESULTADOS}


La concreción de $\mathrm{n}=25$ documentos académicos relacionados con el tema, permite pasar a la fase de una lectura exploratoria y organización de la información. Para lo cual, los artículos se han distribuido en una tabla descriptiva con el propósito de visualizar la información de manera individual en tres categorías como: el citado bibliográfico en base a las normas APA (American Psychological Association), palabras clave y resumen.

Tabla 1 Descripción de la revisión de los estudios

\begin{tabular}{|c|c|c|}
\hline APA & Palabras clave & Resumen \\
\hline $\begin{array}{l}\text { Amador Borrero, M., } \\
\text { Marínez Ferrer, B., } \\
\text { Ávila Guerrero, M. E., } \\
\text { \& Del Moral Arroyo, G. } \\
\text { (2016). Migración } \\
\text { interna de mujeres } \\
\text { náhuatl: perspectiva de } \\
\text { las hijas adolescentes. } \\
\text { Revista Internacional } \\
\text { de Estudios } \\
\text { Migratorios, 6(1), 1-19 }\end{array}$ & $\begin{array}{l}\text { Adolescentes, } \\
\text { percepción } \\
\text { migración, } \\
\text { indígena, } \\
\text { cualitativo }\end{array}$ & $\begin{array}{l}\text { La migración interna de mujeres indígenas en México se } \\
\text { produce por múltiples motivos, uno de ellos es asegurar a sus } \\
\text { hijos e hijas un futuro mejor. Por ello, con este trabajo } \\
\text { pretendemos analizar la experiencia migratoria desde la } \\
\text { perspectiva de las hijas de estas mujeres. }\end{array}$ \\
\hline $\begin{array}{l}\text { Zago, N. (2016). } \\
\text { Migração rural-urbana, } \\
\text { juventude e ensino } \\
\text { superior. } \\
\text { Brasileira de Educação, } \\
21(64), 61-78 .\end{array}$ & $\begin{array}{l}\text { enseñanza superior; } \\
\text { migración rural- } \\
\text { urbana; } \\
\text { juventud y } \\
\text { educación }\end{array}$ & $\begin{array}{l}\text { En Brasil, la proporción entre la población rural y la urbana fue } \\
\text { alterada de modo significativo en las últimas décadas (menos } \\
\text { del } 20 \% \text { vive en el medio rural) y los movimientos migratorios } \\
\text { en dirección a la ciudad son formados por un público más } \\
\text { joven y más femenino que en el pasado. El presente artículo } \\
\text { trata de las relaciones entre las transformaciones sociales, las } \\
\text { demandas por instrucción superior y los destinos socio- } \\
\text { profesionales de los hijos y las hijas de pequeños productores } \\
\text { rurales. }\end{array}$ \\
\hline $\begin{array}{l}\text { De Guevara, L. C. P. L., } \\
\text { López, L. I. C., \& } \\
\text { Pacheco, J. A. M. } \\
\text { (2016). } \\
\text { Interculturalidad y } \\
\text { derecho a la educación } \\
\text { de la niñez indígena } \\
\text { jornalera migrante. } \\
\text { LiminaR. Estudios } \\
\text { Sociales y }\end{array}$ & $\begin{array}{l}\text { Educación } \\
\text { intercultural, } \\
\text { migración, } \\
\text { trabajo infantil, } \\
\text { políticas educativas. }\end{array}$ & $\begin{array}{l}\text { Niños y niñas de diversos pueblos indígenas trabajan en los } \\
\text { campos agrícolas de acuerdo con su edad y género, mientras } \\
\text { que deberían estar en la escuela recibiendo educación de } \\
\text { calidad en su propia lengua. En este artículo se analizan las } \\
\text { acciones en torno a la educación intercultural dirigida a esta } \\
\text { población a partir del análisis de las acciones establecidas por } \\
\text { parte del sector educativo. }\end{array}$ \\
\hline $\begin{array}{l}\text { Aquino-Moreschi, A., \& } \\
\text { Contreras-Pastrana, I. } \\
\text { (2016). Comunidad, } \\
\text { jóvenes y generación: } \\
\text { disputando } \\
\text { subjetividades en la } \\
\text { Sierra Norte de Oaxaca. } \\
\text { Revista } \\
\text { Latinoamericana de } \\
\text { Ciencias Sociales, Niñez } \\
\text { y Juventud, 14(1). }\end{array}$ & $\begin{array}{l}\text { Jóvenes, población } \\
\text { indígena, } \\
\text { migración, } \\
\text { identidad }\end{array}$ & $\begin{array}{l}\text { En este artículo analizamos desde una perspectiva } \\
\text { etnográfica, de qué forma se construye y experimenta la } \\
\text { juventud en diferentes comunidades ayuujk y zapotecas de la } \\
\text { Sierra Norte de Oaxaca, México. En el centro del análisis } \\
\text { ubicamos los conflictos generacionales por la definición de } \\
\text { subjetividades, en particular, las tensiones que tienen que ver } \\
\text { con el cuerpo, las identidades y las apuestas de vida } \\
\text { individuales y colectivas }\end{array}$ \\
\hline
\end{tabular}




\begin{tabular}{|c|c|c|}
\hline $\begin{array}{l}\text { Caqueo-Urízar, A., } \\
\text { Urzúa, A., Ferrer, R., } \\
\text { Pereda, N., Villena, C., } \\
\text { \& Irarrázaval, M. } \\
\text { (2014). Afrontamiento } \\
\text { y etnia: Estrategias en } \\
\text { niños y niñas aymara. } \\
\text { Terapia psicológica, } \\
\text { 32(2), 79-86. }\end{array}$ & $\begin{array}{l}\text { Afrontamiento } \\
\text { Etnia, } \\
\text { Aymara, } \\
\text { Estrés por } \\
\text { aculturación. }\end{array}$ & $\begin{array}{l}\text { Menores aymara han experimentado un proceso migratorio } \\
\text { importante durante las últimas décadas, sin embargo, se han } \\
\text { desarrollado escasos estudios en población infantil } \\
\text { perteneciente a esta etnia. El objetivo de esta investigación es } \\
\text { evaluar y comparar las estrategias de afrontamiento utilizado } \\
\text { por niños y niñas Aymara y no Aymara. }\end{array}$ \\
\hline $\begin{array}{l}\text { Lin, X. (2014). 'Filial } \\
\text { son', the family and } \\
\text { identity formation } \\
\text { among male migrant } \\
\text { workers in urban China. } \\
\text { Gender, Place \& } \\
\text { Culture, 21(6), 717- } \\
732 \text {. }\end{array}$ & $\begin{array}{l}\text { men; } \\
\text { family; } \\
\text { filial practice; } \\
\text { masculine identity; } \\
\text { migration; } \\
\text { China }\end{array}$ & $\begin{array}{l}\text { Se explora las narrativas de hombres migrantes rural-urbanos } \\
\text { (internos) con un enfoque particular en el cambio de las } \\
\text { relaciones sociales dentro de la familia, desde la perspectiva } \\
\text { de los hijos de migrantes. El artículo también explora una } \\
\text { comprensión más compleja de la migración rural-urbana }\end{array}$ \\
\hline $\begin{array}{l}\text { Sobrino, J. (2014). } \\
\text { Migración interna y } \\
\text { tamaño de localidad en } \\
\text { México. Estudios } \\
\text { demográficos } \\
\text { urbanos de El Colegio } \\
\text { de México, 29(3), 443- } \\
480 . \\
\text { ISO } 690\end{array}$ & $\begin{array}{l}\text { Migración interna, } \\
\text { movilidad espacial, } \\
\text { ascendente y } \\
\text { descendente, } \\
\text { características } \\
\text { sociodemográficas } \\
\text { de los migrantes. }\end{array}$ & $\begin{array}{l}\text { La migración rural-urbana es una movilidad espacial } \\
\text { ascendente, debido a que el lugar de origen es de menor } \\
\text { tamaño poblacional que el de destino. En México, y por lo } \\
\text { menos desde el año 2000, la migración urbana-urbana es el } \\
\text { flujo preponderante dentro de la migración interna, y en él se } \\
\text { combinan la movilidad espacial ascendente y la descendente } \\
\text { o de lugares de mayor a menor tamaño de población. El } \\
\text { objetivo de este artículo consiste en analizar los flujos } \\
\text { migratorios por tamaño de localidad en los periodos 1995- } \\
2000 \text { y } 2005-2010 \text { para mostrar las características espaciales y } \\
\text { económico-productivas de los lugares de origen y destino, y } \\
\text { los atributos sociodemográficos de los migrantes en términos } \\
\text { de sexo, edad, nivel educativo e ingresos percibidos. El artículo } \\
\text { revela un mayor dinamismo en el volumen de la movilidad } \\
\text { espacial descendente y unas condiciones más adversas en la } \\
\text { inserción al mercado de trabajo de las personas con movilidad } \\
\text { espacial ascendente. }\end{array}$ \\
\hline $\begin{array}{l}\text { León-torres, D. (2014). } \\
\text { Niños, niñas, y } \\
\text { mujeres: Una } \\
\text { amalgama vulnerable. } \\
\text { Revista } \\
\text { Latinoamericana de } \\
\text { Ciencias Sociales, Niñez } \\
\text { y Juventud, 12(1). }\end{array}$ & $\begin{array}{l}\text { Infancia } \\
\text { desfavorecida, } \\
\text { bienestar social, } \\
\text { cuidado del niño, } \\
\text { estudios sobre } \\
\text { mujeres, migración. }\end{array}$ & $\begin{array}{l}\text { El principal resultado de este trabajo es la documentación de } \\
\text { las desventajas estructurales que caracterizan a la migración } \\
\text { interna femenina y de los efectos que estos movimientos de } \\
\text { población ejercen sobre los hijos de las mujeres migrantes. }\end{array}$ \\
\hline $\begin{array}{l}\text { Amavizca-Ruiz, J. R., } \\
\text { Regalado-López, J., } \\
\text { Álvarez-Gaxiola, J. F., } \\
\text { Méndez-Espinoza, J. A., } \\
\text { Mendoza-Robles, R., } \\
\text { Galvanovskis- } \\
\text { Kasparane, A., \& Díaz- } \\
\text { Puente, J. M. (2014). La }\end{array}$ & $\begin{array}{l}\text { migración } \\
\text { internacional, } \\
\text { motivación, } \\
\text { personalidad } \\
\text { migrante. }\end{array}$ & $\begin{array}{l}\text { El artículo aborda la migración de adolescentes en San Mateo } \\
\text { Ozolco para ubicar, desde la teoría de la personalidad } \\
\text { migrante, porqué algunos adolescentes migran y otros no, } \\
\text { bajo las mismas condiciones de marginación y pobreza. Para } \\
\text { ello se analizan los componentes bajo dicha teoría, cuyos } \\
\text { resultados indican que a través de ésta puede hacerse la } \\
\text { diferenciación entre los que desean hacerlo y los que no, ya } \\
\text { que retoma aspectos internos, como personalidad y }\end{array}$ \\
\hline
\end{tabular}




\begin{tabular}{|c|c|c|}
\hline $\begin{array}{l}\text { migración de la } \\
\text { población adolescente } \\
\text { de San Mateo Ozolco, } \\
\text { Puebla. Agricultura, } \\
\text { sociedad y desarrollo, } \\
11(2), 181-199 .\end{array}$ & & $\begin{array}{l}\text { motivación de logro, poder y afiliación, así como aspectos } \\
\text { situacionales de pobreza y marginación. Asimismo, presenta } \\
\text { una estructura que integra diferentes aspectos teóricos del } \\
\text { tema, además de los aportes de los estudios de la migración } \\
\text { adolescente, de variables de motivación para reunirse con la } \\
\text { familia, valores, expectativas y pobreza, entre otros. }\end{array}$ \\
\hline $\begin{array}{l}\text { Roy, D., Lees, M. H., } \\
\text { Palavalli, B., Pfeffer, K., } \\
\text { \& Sloot, M. P. (2014). } \\
\text { The emergence of } \\
\text { slums: A contemporary } \\
\text { view on simulation } \\
\text { models. Environmental } \\
\text { Modelling \& Software, } \\
59,76-90 .\end{array}$ & $\begin{array}{l}\text { Slums } \\
\text { Informal settlement } \\
\text { Agent based } \\
\text { models } \\
\text { Geographic } \\
\text { information } \\
\text { systems } \\
\text { Cellular automata }\end{array}$ & $\begin{array}{l}\text { The existence of slums or informal settlements is common to } \\
\text { most cities of developing countries. Its role as single housing } \\
\text { delivery mechanism has seriously challenged the popular } \\
\text { notion held by policy makers, planners and architects. Today } \\
\text { informality is a paradigm of city making and economic growth } \\
\text { in Africa, Asia and Latin America. This paper discusses the role } \\
\text { of computer simulation models to understand the emergence } \\
\text { and growth of slums in developing countries. We have } \\
\text { identified the key factors influencing the growth of slums and } \\
\text { formulated a standardized set of criteria for evaluating slum } \\
\text { models. }\end{array}$ \\
\hline $\begin{array}{l}\text { Vargas Urrutia, B. } \\
\text { (2013). Retornos a la } \\
\text { educación y migración } \\
\text { rural-urbana en } \\
\text { Colombia. Desarrollo y } \\
\text { Sociedad, (72), 205- } \\
223 .\end{array}$ & $\begin{array}{l}\text { Retornos de la } \\
\text { educación, } \\
\text { migración, ecuación } \\
\text { de Mincer }\end{array}$ & $\begin{array}{l}\text { Con base en los resultados obtenidos, se especula acerca de } \\
\text { los mercados de trabajo, la calidad de la educación y el sesgo } \\
\text { de selección del grupo migrante como algunas de las posibles } \\
\text { explicaciones de los diferenciales entre los grupos urbano, } \\
\text { rural y migrante }\end{array}$ \\
\hline $\begin{array}{l}\text { Ospina, G. I. (2013). } \\
\text { MIGRACIONES CAMPO- } \\
\text { CIUDAD EN EL } \\
\text { MAGREB/" Rural-Urban } \\
\text { Migration in the } \\
\text { Maghreb". UNISCI } \\
\text { Discussion Papers, (31), } \\
69 .\end{array}$ & $\begin{array}{l}\text { Rural-urban } \\
\text { migration, } \\
\text { Agricultural } \\
\text { policies, } \\
\text { Maghreb, Morocco, } \\
\text { Algeria, } \\
\text { Tunisia }\end{array}$ & $\begin{array}{l}\text { Internal migration flows from rural to urban areas have been } \\
\text { considered one of the leading factors in the creation of } \\
\text { regional imbalances where they occurred. The world is } \\
\text { becoming more urban, and this implies that the activities of } \\
\text { the city attract many people, which inevitably come from rural } \\
\text { areas. In the present case, the phenomenon of rural exodus } \\
\text { has been considered as a challenge to all the agricultural } \\
\text { reforms implemented by the Maghreb countries in their short } \\
\text { period of life since independence. The article explains the } \\
\text { results obtained with the reforms and stress that rural-urban } \\
\text { migration flows continue and will continue in the next coming } \\
\text { year }\end{array}$ \\
\hline $\begin{array}{l}\text { Ortega, T., \& Ng, M. J. } \\
\text { (2013). La migración } \\
\text { del campo a las } \\
\text { ciudades. La pobreza y } \\
\text { la exclusión social de } \\
\text { los pobladores de la } \\
\text { colonia Miguel Alemán } \\
\text { en Xalapa, México. }\end{array}$ & $\begin{array}{l}\text { Migración rural- } \\
\text { urbana, } \\
\text { pobreza, } \\
\text { exclusión social, } \\
\text { identidad social, } \\
\text { organización } \\
\text { vecinal. }\end{array}$ & $\begin{array}{l}\text { Este trabajo analiza la migración rural-urbana, en particular el } \\
\text { caso de los habitantes que llegaron a fundar la Colonia Miguel } \\
\text { Alemán en la ciudad de Xalapa. Se hace una descripción de la } \\
\text { situación del campo en México y en Veracruz para poder } \\
\text { entender el continuo éxodo de campesinos a los centros } \\
\text { urbanos. Respecto a los campesinos que llega-ron a la colonia, } \\
\text { ellos han experimentado condiciones de pobreza extrema y de } \\
\text { exclusión social. Para subsistir se han tenido que incorporar a } \\
\text { la organización vecinal y con el tiempo esta gestión incide para } \\
\text { mejor sus condiciones de vida. Por otra parte, se explica cómo } \\
\text { los colonos han ido desarrollando competencias culturales } \\
\text { producto de un proceso de hibridación cultural entre las } \\
\text { pautas de la sociedad rural tradicional con la cultura urbana a } \\
\text { la que se han adaptado. }\end{array}$ \\
\hline
\end{tabular}




\begin{tabular}{|c|c|c|}
\hline $\begin{array}{l}\text { Yang, J. (2013). Social } \\
\text { Exclusion and Young } \\
\text { Rural-Urban Migrants' } \\
\text { Integration into a Host } \\
\text { Society in China. The } \\
\text { ANNALS of the } \\
\text { American Academy of } \\
\text { Political and Social } \\
\text { Science, 648(1), 52-69. }\end{array}$ & $\begin{array}{l}\text { rural-urban } \\
\text { migrants; } \\
\text { urban-urban } \\
\text { migrants; } \\
\text { socioeconomic } \\
\text { status; } \\
\text { Hukou system; } \\
\text { social exclusion; } \\
\text { China }\end{array}$ & $\begin{array}{l}\text { Este artículo explora los correlatos de la integración } \\
\text { socioeconómica de los jóvenes migrantes rural-urbano } \\
\text { Usando una nueva tipología que distingue hukou (sistema de } \\
\text { registro de residencia), el estado de la migración, y la edad, los } \\
\text { resultados del modelo de niveles múltiples indican que los } \\
\text { jóvenes migrantes rural-urbanos lograr un estatus } \\
\text { socioeconómico más bajo que los jóvenes locales y jóvenes } \\
\text { migratorias urbana-urbana. Esto desafía la noción de que la } \\
\text { mercantilización promueve necesariamente derechos y la } \\
\text { igualdad jurídica de forma lineal y sugiere que el impacto } \\
\text { potencialmente positivo de la migración en el desarrollo } \\
\text { personal podría verse comprometida por restricciones } \\
\text { institucionales (por ejemplo, hukou) que excluyen a los } \\
\text { migrantes de las zonas rurales, así como otra extraña, } \\
\text { particularmente jóvenes. }\end{array}$ \\
\hline $\begin{array}{l}\text { Horbath, J. E. (2013). } \\
\text { De la marginación rural } \\
\text { a la exclusión escolar } \\
\text { urbana: el caso de los } \\
\text { niños y jóvenes } \\
\text { indígenas que migran a } \\
\text { las ciudades del sureste } \\
\text { mexicano. Espiral } \\
\text { (Guadalajara), 20(58), } \\
\text { 135-169. }\end{array}$ & $\begin{array}{l}\text { Social exclusion, } \\
\text { rural-urban } \\
\text { migration, } \\
\text { indigenous children } \\
\text { and youth, } \\
\text { multicultural } \\
\text { education; }\end{array}$ & $\begin{array}{l}\text { Los contrastes demográficos en el sureste de México son } \\
\text { notables y muestran los flujos de migración indígena hacia las } \\
\text { principales ciudades de esta región. Los datos censales revelan } \\
\text { que en esas entidades el rezago social ha ido en aumento y el } \\
\text { reemplazo etnolingüístico ha tendido a reducirse, pues los } \\
\text { indígenas instalados en los núcleos urbanos prefieren ocultar } \\
\text { su procedencia étnica y abandonar el uso de su lengua } \\
\text { materna. El sistema educativo urbano no está capacitado para } \\
\text { ofrecer una enseñanza multicultural; al contrario, compele a } \\
\text { los niños y jóvenes indígenas que han migrado con sus padres } \\
\text { a abandonar el uso de su lengua materna. Así, este grupo de } \\
\text { población es sometido a un proceso de exclusión doble: el } \\
\text { primero es el que los obliga a abandonar sus comunidades y el } \\
\text { segundo es el que les impide acceder a la educación en } \\
\text { condiciones favorables. }\end{array}$ \\
\hline $\begin{array}{l}\text { Shrestha, B. K. (2013, } \\
\text { March). Squatter } \\
\text { settlements in the } \\
\text { Kathmandu valley: } \\
\text { Looking through the } \\
\text { prism of land rights and } \\
\text { tenure security. In } \\
\text { Urban Forum (Vol. 24, } \\
\text { No. 1, pp. 119-135). }\end{array}$ & $\begin{array}{l}\text { Slum and squatter } \\
\text { settlements, } \\
\text { Land right, } \\
\text { Tenure security, } \\
\text { Kathmandu, } \\
\text { Valley, } \\
\text { Poverty }\end{array}$ & $\begin{array}{l}\text { La migración rural-urbana ha dado como resultado el rápido } \\
\text { crecimiento de los asentamientos irregulares en el valle de } \\
\text { Katmandú. Esta migración es debido al bajo crecimiento } \\
\text { socioeconómico, un sistema de administración de tierras } \\
\text { pobres, la capacidad insuficiente para hacer frente a las } \\
\text { necesidades de vivienda y el mal gobierno. (...), la falta de } \\
\text { acceso a las opciones de financiación, el aumento de la } \\
\text { vulnerabilidad y la exclusión social. }\end{array}$ \\
\hline 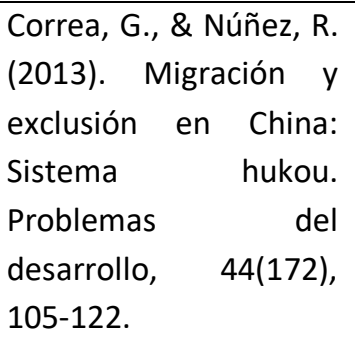 & $\begin{array}{l}\text { China, } \\
\text { migración, } \\
\text { hukou, } \\
\text { bienestar, } \\
\text { desarrollo } \\
\text { económico. }\end{array}$ & $\begin{array}{l}\text { Se destaca en el texto que el notable crecimiento económico } \\
\text { chino de las últimas décadas ha resultado en desigualdad } \\
\text { económica social y cómo a pesar de que las reformas al hukou } \\
\text { aumentaron la movilidad de la población, para los migrantes } \\
\text { tanto rurales como residentes urbanos de otras ciudades se } \\
\text { mantienen las dificultades de acceso a beneficios y servicios } \\
\text { públicos por estar registrados en otro lugar. }\end{array}$ \\
\hline $\begin{array}{l}\text { Pérez Campuzano, E., \& } \\
\text { Santos Cerquera, C. } \\
\text { (2013). Tendencias } \\
\text { recientes de la }\end{array}$ & $\begin{array}{l}\text { Migración interna, } \\
\text { México, }\end{array}$ & $\begin{array}{l}\text { Hay evidencia de la transición de la migración interna en } \\
\text { países en desarrollo. El patrón tradicional rural-urbano ha } \\
\text { evolucionado hacia uno de tipo urbano-urbano, mismo que } \\
\text { indica la transformación de otros importantes indicadores: }\end{array}$ \\
\hline
\end{tabular}




\begin{tabular}{|c|c|c|}
\hline $\begin{array}{l}\text { migración interna en } \\
\text { México. Papeles de } \\
\text { población, 19(76), 53- } \\
88 .\end{array}$ & $\begin{array}{l}\text { urbanización, } \\
\text { desconcentración } \\
\text { de la población. }\end{array}$ & $\begin{array}{l}\text { desarrollo urbano-regional, el incremento de las } \\
\text { deseconomías de aglomeración en las principales ciudades, } \\
\text { entre otros }\end{array}$ \\
\hline $\begin{array}{l}\text { Aparicio, P., \& Ruiz, F. } \\
\text { (2012). Jóvenes, } \\
\text { migración y procesos } \\
\text { de integración } \\
\text { socioeducativa en } \\
\text { Argentina. La ruralidad } \\
\text { como estigma y } \\
\text { fractura de la cohesión } \\
\text { social. Revue } \\
\text { interdisciplinaire des } \\
\text { travaux sur les } \\
\text { Amériques, (5), 1-20. }\end{array}$ & $\begin{array}{l}\text { Juventud, } \\
\text { Educación, } \\
\text { Ruralidad, } \\
\text { Migración, } \\
\text { Participación social. }\end{array}$ & $\begin{array}{l}\text { En América latina y particularmente en Argentina, las y los } \\
\text { jóvenes que viven en contextos rurales están más expuestos a } \\
\text { la vulnerabilidad económica, la exclusión social y la pobreza } \\
\text { que sus pares urbanos. En este contexto, la migración se } \\
\text { presenta como una alternativa para amedrentar el impacto de } \\
\text { las restricciones sociales y económicas }\end{array}$ \\
\hline $\begin{array}{l}\text { Jurado, C., \& Tobasura, } \\
\text { I. (2012). Dilema de la } \\
\text { juventud en territorios } \\
\text { rurales de Colombia:¿ } \\
\text { campo o ciudad?. } \\
\text { Revista } \\
\text { Latinoamericana de } \\
\text { Ciencias Sociales, niñez } \\
\text { y juventud, 10(1), 63- } \\
77 .\end{array}$ & $\begin{array}{l}\text { juventud rural, } \\
\text { migración rural, } \\
\text { cultural identidad, } \\
\text { entorno social }\end{array}$ & $\begin{array}{l}\text { En este artículo presentamos algunos resultados del estudio } \\
\text { "Experiencias alternativas de acción política con participación } \\
\text { de jóvenes", trabajo en el que se busca comprender el modo } \\
\text { en que las transiciones demográficas, migratorias y } \\
\text { productivas contribuyen a la construcción de las identidades } \\
\text { juveniles y de sus proyectos de vida, en entornos rurales del } \\
\text { Eje Cafetero e identificar las motivaciones de los jóvenes y de } \\
\text { las jóvenes, sus problemas identitarios, sus expectativas y } \\
\text { algunas de las razones para irse del campo. El trabajo nos } \\
\text { permite concluir que, aunque el ámbito rural tiende a ser bien } \\
\text { valorado como espacio de vida, las expectativas laborales y } \\
\text { familiares y la identidad de los sujetos jóvenes parece } \\
\text { construirse sobre el imaginario de la ciudad. }\end{array}$ \\
\hline $\begin{array}{l}\text { Urteaga Castro Pozo, } \\
\text { M. (2011). Retos } \\
\text { contemporáneos en los } \\
\text { estudios } \quad \text { sobre } \\
\text { juventud. Alteridades, } \\
21(42), 13-32 .\end{array}$ & $\begin{array}{l}\text { juventudes } \\
\text { indígenas, } \\
\text { migración, paisajes } \\
\text { étnicos juveniles, } \\
\text { zonas fronterizas, } \\
\text { jóvenes indígenas } \\
\text { rurales, } \\
\text { jóvenes indígenas } \\
\text { migrantes en la } \\
\text { ciudad }\end{array}$ & $\begin{array}{l}\text { El texto explora las formas de construcción juvenil étnicas; } \\
\text { indaga las razones por las que la etnografía clásica invisibilizó } \\
\text { al sujeto joven al interior de las etnias; revela las } \\
\text { transformaciones sociales y teóricas por las que la literatura } \\
\text { reciente lo ha visibilizado, proponiendo una línea teórica y } \\
\text { metodológica para abordar a los jóvenes en los nuevos } \\
\text { contextos étnicos; profundiza en la especificidad de lo juvenil } \\
\text { indígena rural y lo juvenil indígena }\end{array}$ \\
\hline $\begin{array}{l}\text { Caballeros, Á. (2011). } \\
\text { Migración con rostro } \\
\text { de niños, niñas y } \\
\text { adolescentes. } \\
\text { Encuentro: Revista } \\
\text { Académica de la } \\
\text { Universidad } \\
\text { Centroamericna, (90), } \\
\text { 84-93. }\end{array}$ & $\begin{array}{l}\text { Niñez migrante, } \\
\text { deportación, } \\
\text { Derechos Humanos }\end{array}$ & $\begin{array}{l}\text { Análisis descriptivo de los niños, niñas y adolescentes } \\
\text { migrantes no acompañados que fueron deportados y } \\
\text { atendidos por el Hogar Casa Nuestras Raíces de la Secretaría } \\
\text { de Bienestar Social de la Secretaría de Bienestar Social de } \\
\text { Guatemala. }\end{array}$ \\
\hline $\begin{array}{l}\text { Unda Lara, R., \& } \\
\text { Muñoz, G. (2011). La }\end{array}$ & $\begin{array}{l}\text { Comunidad, } \\
\text { Indígena, }\end{array}$ & $\begin{array}{l}\text { El presente artículo es producto de una investigación en curso } \\
\text { en tres provincias de la Sierra Central del Ecuador en que se }\end{array}$ \\
\hline
\end{tabular}




\begin{tabular}{|c|c|c|}
\hline $\begin{array}{l}\text { condición juvenil } \\
\text { indígena: elementos } \\
\text { iniciales para su } \\
\text { construcción } \\
\text { conceptual. Última } \\
\text { década, 19(34), 33-50. }\end{array}$ & Jóvenes & $\begin{array}{l}\text { analizan tres procesos fundamentales que intervienen el } \\
\text { espacio de las dinámicas comunales del mundo indígena } \\
\text { andino: a) relaciones (neo) coloniales cifradas en el nombrar } \\
\text { los sujetos y sus relaciones, en el instituir prácticas y en el } \\
\text { administrar población; b) la relación con la tierra y sus distintas } \\
\text { morfologías de reproducción socioeconómica y simbólica; c) la } \\
\text { movilidad humana expresada en los procesos migratorios. }\end{array}$ \\
\hline $\begin{array}{l}\text { Ramos, D. V. (2011). } \\
\text { Migración, niñez y } \\
\text { educación como } \\
\text { procesos culturales. } \\
\text { Una mirada al caso } \\
\text { costarricense: } \\
\text { develando lo oculto } \\
\text { para transformarlo. } \\
\text { ÍsTMICA. Revista de la } \\
\text { Facultad de Filosofía y } \\
\text { Letras, (14), 95-110. }\end{array}$ & $\begin{array}{l}\text { Niñez, } \\
\text { Adolescencia, } \\
\text { Migración } \\
\text { Sociedad } \\
\text { costarricense, } \\
\text { Educación } \\
\text { costarricense }\end{array}$ & $\begin{array}{l}\text { A lo largo de la historia hay múltiples ejemplos donde la } \\
\text { educación ha estado cerrada para el disfrute de ciertos grupos } \\
\text { humanos. Las personas menores de edad, en particular } \\
\text { cuando son inmigrantes, experimentan despojos y } \\
\text { obliteraciones cotidianas, formal o informalmente, en la } \\
\text { práctica de sus derechos, incluido el de equidad en la } \\
\text { educación. Este ensayo ofrece prolegómenos sobre la } \\
\text { construcción del concepto niñez, para luego reflexionar sobre } \\
\text { el caso costarricense, particularmente sobre las percepciones } \\
\text { de educación, migración, niñez y adolescencia. }\end{array}$ \\
\hline $\begin{array}{l}\text { Alvarado, B. (2010). Del } \\
\text { campo a la ciudad: } \\
\text { experiencias sobre } \\
\text { migración y trabajo } \\
\text { doméstico } \\
\text { adolescentes rurales. } \\
\text { Revista venezolana de } \\
\text { estudios de la mujer, } \\
\text { 15(35), 117-133. }\end{array}$ & $\begin{array}{l}\text { migración } \\
\text { femenina, } \\
\text { trabajo } \\
\text { infantil, } \\
\text { Perú, } \\
\text { educación. }\end{array}$ & $\begin{array}{l}\text { En América Latina, el trabajo doméstico es una actividad } \\
\text { predominantemente urbana realizada por mujeres, quienes } \\
\text { en su mayoría abandonan los campos de cultivo para migrar a } \\
\text { las ciudades. El trabajo doméstico está regulado de acuerdo a } \\
\text { normas de los países de la región; sin embargo, las } \\
\text { trabajadoras domésticas, en su mayoría adolescentes y niñas, } \\
\text { aún se encuentran al margen de los derechos laborales y en } \\
\text { total vulnerabilidad. } \\
\text { El presente estudio cualitativo describe las experiencias de un } \\
\text { grupo de adolescentes como trabajadoras domésticas en } \\
\text { centros urbanos del Perú. Los resultados del mismo sugieren } \\
\text { el fortalecimiento de los agentes sociales y de políticas } \\
\text { públicas que aseguren los derechos laborales a este grupo } \\
\text { vulnerable. }\end{array}$ \\
\hline $\begin{array}{l}\text { Tacoli, C., \& Mabala, R. } \\
\text { (2010). Exploring } \\
\text { mobility and migration } \\
\text { in the context of rural- } \\
\text { urban linkages: why } \\
\text { gender and generation } \\
\text { matter. Environment } \\
\text { and Urbanization, } \\
22(2), 389-395 .\end{array}$ & $\begin{array}{l}\text { Gender, } \\
\text { Generation, } \\
\text { Livelihoods, } \\
\text { Migration, } \\
\text { rural-urban } \\
\text { linkages }\end{array}$ & $\begin{array}{l}\text { This paper draws on case studies in Mali, Nigeria, Tanzania and } \\
\text { Vietnam to explore the different ways in which migration } \\
\text { intersects with the changing relations between rural and } \\
\text { urban areas and activities, and in the process transforms } \\
\text { livelihoods and the relations between young and older men } \\
\text { and women. Livelihood strategies are becoming increasingly } \\
\text { diverse, and during interviews people were asked to describe } \\
\text { their first, second and third occupations, the time allocated to } \\
\text { each and the income that each produced. In all study regions, } \\
\text { the number of young people migrating is increasing. This is } \\
\text { influenced not only by expanding employment opportunities } \\
\text { in destination areas but also by power inequalities within } \\
\text { households, which means limited opportunities at home. }\end{array}$ \\
\hline
\end{tabular}

Organizada la documentación en la matriz, se ha proseguido con el análisis, reflexión, discusión e interpretación de la información (Cué Brugueras, Díaz Alonso, Díaz Martínez, Valdés Abreu, 2008). En esta fase las acciones consideradas son: revisión en conjunto, triangulación de información (ejes temáticos, 
los resultados y la discusión) que presentan cada documento y las tendencias e interpretaciones de los investigadores. En el siguiente apartado se presenta la estructura y se define las categorías perfiladas.

\section{Migración rural-urbana}

La migración es un fenómeno complejo, dinámico y multifacético que abarca, una amplia gama de formas y tipos de movimiento, un creciente número de destinos y flujos migratorios con diversa composición étnica, sexo y edad (Tacoli \& Mabala, 2010). Generalmente este proceso de migración presenta dos momentos: la emigración y la inmigración la primera evoca desde un punto de partida, y la segunda el de llegada (Pérez \& Santos, 2013). Frecuentemente suele diferenciarse entre migración interna (desplazamiento dentro del país) e internacional (entre países) (Amador, Marínez, Ávila \& Del Moral, 2016). Desde los inicios de la industrialización la migración interna estuvo marcada por desplazamientos "rural-urbanos" (Pérez \& Santos, 2013; Sobrino, 2013; Vargas, 2013; Zago, 2016).

La migración rural-urbana se da con características propias según el medio rural que rodea a la población (Ortega \& Ng, 2013, Zago, 2016) y termina proyectándose en los procesos de urbanización sustentada sobre la base de un crecimiento incesante de población que llegan de espacios rurales a las ciudades (Pérez \& Santos, 2013). En las últimas décadas varios investigadores señalan, que este tipo de movimiento migratorio rural-urbana o del campo a la ciudad son representados por una población más joven y más femenino que los años pasados (Tacoli \& Mabala, 2010; Ortega \& Ng, 2013; Horbath, 2013; Sobrino, 2013; Lin, 2014; Zago, 2016; Amador, Marínez, Ávila \& Del Moral, 2016). La migración de las personas adolescentes y jóvenes habitantes de territorios rurales, se presenta como "un proceso de identidad contradictorio de apropiaciones, expropiaciones y resignificaciones de proyectos de vida juveniles en dichos escenarios de vida, que contiene, hoy más que nunca, oposiciones y rupturas, antes que una linealidad y un producto estático e inmutable" (Jurado \& Tobasura, 2012, p. 74 ). Probablemente la mayor disyuntiva que enfrenta esta población está relacionada con el aumento de flujos familiares, oportunidades educativas y una búsqueda de mejores condiciones de vida (Ramos, 2011; Urteaga, 2011; Jurado \& Tobasura, 2012; Ortega \& Ng, 2013; Amador, Marínez, Ávila \& Del Moral, 2016).

\section{Causas de la migración rural - urbana}

Las causas de la migración rural-urbana protagonizada por los jóvenes y adolescentes son múltiples, interdependientes y estrechamente relacionadas con las teorías de expulsión y atracción (Amavizca et al, 2014). Por lo que una primera causa se atribuye a situaciones sociales, económicas, educativos, culturales, políticos y fenómenos naturales (Aparicio \& Ruiz, 2012). La segunda se le suma la búsqueda de mejoras en la calidad de vida y salir adelante que son invariables presentes en la cotidianidad del ser humano (Ramos, 2011; Ortega \& Ng, 2013; Amador, Marínez, Ávila \& Del Moral, 2016; Aparicio \& Ruiz, 2012). Desplegando lo anterior Ortega \& Ng (2013), Amavizca, et al, (2014) identifican que la decisión de migrar del campo a la ciudad se relaciona con factores estructurales, tales como pobreza, marginación, violencia y exclusión. Del mismo modo, Jurado \& Tobasura (2012), Aparicio \& Ruiz (2012) argumentan que la opción de migrar por los sujetos jóvenes tiende a ser considerada por la falta de opciones laborales y privación del acceso a oportunidades educativas y sociales. Además, Caballeros (2011) señala que los niños y adolescentes migran por tres motivos búsqueda de trabajo, reunificación familiar y paseo. Otra causa de la migración del campo a la ciudad se apoya en las catástrofes ambientales, reducción de tierras, escasa productividad agropecuaria, la falta de apoyo al campo agrícola y los altos estándares de exportación que imponen los Tratados de Libre Comercio (Correa \& Nuñez, 2013; Ortega \& Ng, 2013; Zago; 2016). Estas fuentes según Urteaga (2011) dan lugar a dos tipos de migración de jóvenes y adolescentes rurales: a) los migrantes rural-urbanos que buscan trabajo, constituido por jóvenes y adolescentes, que "salen en busca de trabajo" por las vías industriales del centro del país (cargadores, limpia parabrisas, vendedores de la calle, 
o empleados y obreros en fábricas) (Ortega \& Ng, 2013). b) Los migrantes rural-urbanos que buscan educación, son los jóvenes y adolescentes que "migran para estudiar" (escuela, educación superior) con apoyo de algún familiar o combinando trabajo y estudio en diferentes ciudades (Zago, 2016).

Sin embargo, en el proceso de la migración rural - urbano, se presenta resultados positivos y negativas. Dentro de los márgenes positivos encontramos la disminución de la presión sobre los recursos naturales, la infraestructura y los presupuestos públicos, participación en la movilidad social, posibilidades de tener mejores empleos y condiciones de una vida mejor a largo plazo (Correa \& Nuñez, 2013; Ortega \& $\mathrm{Ng}$, 2013). Como contrapartida se ve reflejado en un sentimiento de pérdida de identidad (Horbath, 2013) nacional y costumbres. Decaimiento de rendimiento y deserción escolar, trabajos con bajos niveles salariales, empleos precarios, crecimiento del sector informal, conductas desviadas, colapsos ambientales (Ortega \& Ng, 2013; Aparicio \& Ruiz, 2012). Así como la ruptura con lazos familiares y amistades.

\section{Migración Rural-Urbana Y Exclusión Social}

La exclusión social hace referencia al "proceso por el cual los individuos y comunidades enteras de personas, incluidos los migrantes, son sistemáticamente bloqueadas de los derechos, oportunidades y recursos, factores claves de la integración socioeconómica, que normalmente están disponibles para los miembros de la sociedad" (Yang, 2013, p. 55). Este proceso a menudo es conceptualizado como dinámico, multidimensional y flexible, lo que permite un mejor análisis de las sociedades complejas (Ortega \& $\mathrm{Ng}$, 2013; Yang, 2013). Los excluidos en una sociedad heterogénea son separados de las relaciones e instituciones y limitados en la participación de actividades sociales (Yang, 2013). Bajo esta perspectiva el proceso migratorio se constituye como nuevas formas, dinámicas y espacios de inclusión y exclusión social (Aparicio \& Ruiz, 2012, p. 6). Los flujos de migración rural-urbana crecen intensamente en diferentes países del mundo a pesar de las malas condiciones en los barrios pobres y exclusión social (Horbath, 2013; Shrestha, 2013; Ortega \& Ng, 2013; Yang, 2103; Roy, Lees, Palavalli, Pfeffer \& Sloot, 2014). La mayor parte de esta población comparte características de marginación, condiciones de extrema pobreza, analfabetismo y discriminación (Horbath, 2013; Ortega \& Ng, 2013). En medio de esta gran escala de migración rural-urbana, los jóvenes y adolescentes se han convertido en uno de los segmentos más vulnerables en diferentes regiones (Caballeros, 2011; Yang, 2013; Zago, 2016). La discriminación urbana a los jóvenes y adolescentes migrantes es aguda y de tanto arraigo deja secuelas claras en su autoestima, provocando a que ellos mismos se discriminen (Horbath, 2013).

Los jóvenes y adolescentes migrantes del campo a la ciudad, son expuestos a la incertidumbre económica (Horbath, 2013; Aparicio \& Ruiz, 2012) y a múltiples problema sociales de riesgo tales como alcoholismo, drogadicción, vandalismo, prostitución y violencia (Shrestha, 2013; Jurado \& Tobasura, 2012). En las ciudades instalados obtienen acceso restringido a servicios públicos de seguridad social y derechos que otorga el gobierno (Ortega \& Ng, 2013). Son parte del mercado laboral con malas condiciones salariales y contratos temporales de trabajo o escasas prestaciones laborales, a menudo acuden a servicios prestados por particulares que generalmente están fuera de las regulaciones locales (Corre \& Nuñez, 2012). La desigualdad sobresale en términos de acceso a servicios de educación, salud, patrimonio, vivienda y el retiro de las políticas públicas que determinan el bienestar de esta población (Correa \& Nuñez, 2013; Ortega $\& \mathrm{Ng}, 2103$; Horbath, 2013). Son pobres que viven en el abandono psicológico y social (Ortega \& Ng, 2013). Esto genera procesos que impiden a los jóvenes y adolescentes migrantes, la posibilidad de una subsistencia autónoma en los rangos determinados por las instituciones (Jurado \& Tobasura, 2012).

\section{Factores de riesgo de exclusión social y educativa}

La exclusión social se manifiesta en función de diferentes factores de riesgo que se interrelacionan entre sí y varia de un grupo a otro. No se puede entender y explicar considerando un único factor, ni una 
única dimensión, por lo que se dice que la exclusión social se presenta de forma multidimensional y multifactorial (Yang, 2013). En este sentido, en las investigaciones de Jurado \& Tobasura (2012), Ortega \& Ng (2013), Yang (2013), Shrestha (2013), Aparicio \& Ruiz (2012), De Guevara, López \& Pacheco (2016) y otros, se identifica y determinan diferentes factores de exclusión a los que son sometidos los jóvenes y adolescentes que migran del campo a la ciudad. Para su mejor visualización se agrupa y presenta en una tabla:

Tabla 2 Dimensiones y factores de riesgo de exclusión social

\begin{tabular}{|l|l|}
\hline Dimensiones & Factores de riesgo \\
\hline Educativos/Formativos & $\begin{array}{l}\text { Segregación educativa } \\
\text { Inasistencia escolar } \\
\text { Fracaso escolar } \\
\text { Analfabetismo } \\
\text { Deserción escolar }\end{array}$ \\
\hline Laborales & $\begin{array}{l}\text { Empleo precario } \\
\text { Explotación de mano de obra barata } \\
\text { Inestabilidad laboral } \\
\text { Comercio informal (vendedores de la calle) } \\
\text { Explotación infantil }\end{array}$ \\
\hline Familiares & $\begin{array}{l}\text { Sin redes sociales } \\
\text { Carencia de vivienda, red vial, servicios básicos }\end{array}$ \\
\hline Culturales & $\begin{array}{l}\text { Diferencias culturales, costumbres. } \\
\text { Negación y prejuicios culturales de origen } \\
\text { Negación de procedencia étnica } \\
\text { Abandono del uso de la lengua materna } \\
\text { Pérdida de identidad } \\
\text { Mimetización }\end{array}$ \\
\hline Políticos & $\begin{array}{l}\text { Discriminaciones en acceso a determinados lugares } \\
\text { Bajo o nulos niveles de cobertura de seguridad social y salud } \\
\text { Conductas desviadas (delincuencia, drogadicción, alienación). }\end{array}$ \\
& $\begin{array}{l}\text { Carencia de derecho a voto } \\
\text { Insuficientes apoyos de los programas del gobierno. } \\
\text { Vivienda restringida }\end{array}$ \\
& \\
\hline & \\
& \\
&
\end{tabular}

La tabla 2, muestra los factores de riesgo generadores de exclusión que se concretan en diferentes ámbitos vitales de la vida de los adolescentes y jóvenes migrantes instalados en núcleos urbanos (Ortega \& $\mathrm{Ng}, 2013)$. En esa concreción de factores o variables interrelacionados que desencadenan procesos excluyentes de precariedad laboral y habitacional, rezago social y pérdida de identidad en la ciudad (Horbath, 2013), destacamos la dimensión educativa.

La educación, que representa una oportunidad para alcanzar calidad de vida, no está plenamente asegurada en las dinámicas de migración de jóvenes y adolescentes. Como revelan los estudios, la expectativa educativa es uno de los "ámbitos de mayor tensión y conflicto intercultural" debido a las prácticas de marginación en las instituciones, discriminación por parte de los directivos escolares, docentes (que 
prohíben comunicarse en lenguas nativas) y estudiantes mestizos (Alvarado, 2010; Urteaga, 2011, p. 24, Horbarth, 2013). En este ambiente de vulnerabilidad social, la población de jóvenes y adolescentes migrantes están expuestos a mayores probabilidades de inasistencia o abandono escolar, analfabetismo funcional, carencia de credenciales educativa satisfactorias y atractivas (Aparicio \& Ruiz, 2012, p.4). Y en situaciones donde la exclusión va más allá del aspecto laboral y económico de sus padres, deciden no acudir y participar a ningún tipo de educación (Ortega \& Ng, 2013). Puesto que comparten o se integran al comercio informal o a extenuantes jornadas de trabajo para generar ingresos y apoyar la economía familiar (Alvarado, 2010; Jurado \& Tobasura, 2012). En ese sentido la educación, un aspecto importante en la construcción de sus vidas pasa a un segundo plano.

\section{CONCLUSIONES}

La literatura revisada permite concluir que la migración es un fenómeno complejo y dinámico. En esta medida la comunidad de migrantes de territorios rurales a urbanos es representado en su mayoría por una población joven y femenina. Diversas son las causas por la que los jóvenes y adolescentes se desplazan. Más de una se atribuye a situaciones sociales, económicas, educativos, culturales, políticos y fenómenos naturales. Y la búsqueda de mejores condiciones de vida. Sin embargo, gran parte de jóvenes y adolescentes que se van instalando en las ciudades son sometidos a procesos dinámicos de exclusión social.

La exclusión social hace referencia al bloqueo de los derechos, oportunidades y recursos a los individuos y comunidades enteras de personas. En ese sentido las investigaciones reportan que el colectivo de jóvenes y adolescentes migrantes del campo a la ciudad son bloqueados al acceso de calidad de servicios públicos y de seguridad social. Así mismo son sometidos al mercado laboral con malas condiciones salariales y contratos temporales de trabajo, rezago social y pérdida de identidad en la ciudad. Estos factores de riesgo de exclusión son agrupados en términos educativos, laborales, familiares, políticos y culturales. En la interrelación y concreción de los factores de riesgo la dimensión educativa es uno de los "ámbitos de mayor tensión y conflicto intercultural" debido a la existencia de prácticas de marginación y discriminación en las instituciones educativas. Tales prácticas de exclusión conllevan a los jóvenes y adolescentes migrantes a mayores probabilidades de inasistencia o abandono escolar, analfabetismo funcional o fracaso escolar.

\section{REFERENCIAS BIBLIOGRÁFICAS}

Alvarado, B. (2010). Del campo a la ciudad: experiencias sobre migración y trabajo doméstico en adolescentes rurales. Revista venezolana de estudios de la mujer, 15(35), 117-133.

Amador Borrero, M., Marínez Ferrer, B., Ávila Guerrero, M. E., \& Del Moral

Aquino-Moreschi, A., \& Contreras-Pastrana, I. (2016). Comunidad, jóvenes y generación: disputando subjetividades en la Sierra Norte de Oaxaca. Revista Latinoamericana de Ciencias Sociales, Niñez y Juventud, 14(1).

Amavizca-Ruiz, J. R., Regalado-López, J., Álvarez-Gaxiola, J. F., Méndez-Espinoza, J. A., MendozaRobles, R., Galvanovskis-Kasparane, A., \& Díaz-Puente, J. M. (2014). La migración de la población adolescente de San Mateo Ozolco, Puebla. Agricultura, sociedad y desarrollo, 11(2), 181-199.

Aparicio, P., \& Ruiz, F. (2012). Jóvenes, migración y procesos de integración socioeducativa en Argentina. La ruralidad como estigma y fractura de la cohesión social. Revue interdisciplinaire des travaux sur les Amériques, (5), 1-20.

Arroyo, G. (2016). Migración interna de mujeres náhuatl: perspectiva de las hijas adolescentes. Revista Internacional de Estudios Migratorios, 6(1), 1-19

Caballeros, Á. (2011). Migración con rostro de niños, niñas y adolescentes. Encuentro: Revista Académica de la Universidad Centroamericna, (90), 84-93.

Caqueo-Urízar, A., Urzúa, A., Ferrer, R., Pereda, N., Villena, C., \& Irarrázaval, M. (2014). Afrontamiento y etnia: Estrategias en niños y niñas aymara. Terapia psicológica, 32(2), 79-86. 
Correa, G., \& Núñez, R. (2013). Migración y exclusión en China: Sistema hukou. Problemas del desarrollo, 44(172), 105-122.

Cué Brugueras, M., Díaz Alonso, G., Díaz Martínez, A. G., \& Valdés Abreu, M. D. L. C. (2008). El artículo de revisión. Revista Cubana de Salud Pública, 34(4), 0-0.

De Guevara, L. C. P. L., López, L. I. C., \& Pacheco, J. A. M. (2016). Interculturalidad y derecho a la educación de la niñez indígena jornalera migrante. LiminaR. Estudios Sociales

Horbath, J. E. (2013). De la marginación rural a la exclusión escolar urbana: el caso de los niños y jóvenes indígenas que migran a las ciudades del sureste mexicano. Espiral (Guadalajara), 20(58), 135-169.

Jurado, C., \& Tobasura, I. (2012). Dilema de la juventud en territorios rurales de Colombia: ¿campo o ciudad?. Revista Latinoamericana de Ciencias Sociales, niñez y juventud, 10(1), 63-77.

León-torres, D. (2014). Niños, niñas, y mujeres: Una amalgama vulnerable. Revista Latinoamericana de Ciencias Sociales, Niñez y Juventud, 12(1).

Lin, X. (2014). 'Filial son', the family and identity formation among male migrant workers in urban China. Gender, Place \& Culture, 21(6), 717-732.

Morales, H. S., \& Rosario, M. (2012). En los límites de la exclusión social. Inmigración y" sinhogarismo" en España. Papers: revista de sociología, 97(4), 0829-847.

Roy, D., Lees, M. H., Palavalli, B., Pfeffer, K., \& Sloot, M. P. (2014). The emergence of slums: A contemporary view on simulation models. Environmental Modelling \& Software, 59, 76-90.

Sobrino, J. (2014). Migración interna y tamaño de localidad en México. Estudios demográficos y urbanos de El Colegio de México, 29(3), 443-480. ISO 690

Ospina, G. I. (2013). Migraciones Campo-Ciudad En El Magreb/" Rural-Urban Migration in the Maghreb". UNISCI Discussion Papers, (31), 69.

Ortega, T., \& Ng, M. J. (2013). La migración del campo a las ciudades. La pobreza y la exclusión social de los pobladores de la colonia Miguel Alemán en Xalapa, México.

Pérez Campuzano, E., \& Santos Cerquera, C. (2013). Tendencias recientes de la migración interna en México. Papeles de población, 19(76), 53-88.

Ramos, D. V. (2011). Migración, niñez y educación como procesos culturales. Una mirada al caso costarricense: develando lo oculto para transformarlo. ÍSTMICA. Revista de la Facultad de Filosofía y Letras, (14), 95-110.

Shrestha, B. K. (2013, March). Squatter settlements in the Kathmandu valley: Looking through the prism of land rights and tenure security. In Urban Forum (Vol. 24, No. 1, pp. 119-135).

Tacoli, C., \& Mabala, R. (2010). Exploring mobility and migration in the context of rural — urban linkages: why gender and generation matter. Environment and Urbanization, 22(2), 389-395.

Urteaga Castro Pozo, M. (2011). Retos contemporáneos en los estudios sobre juventud. Alteridades, 21(42), 13-32.

Unda Lara, R., \& Muñoz, G. (2011). La condición juvenil indígena: elementos iniciales para su construcción conceptual. Última década, 19(34), 33-50.

Vargas Urrutia, B. (2013). Retornos a la educación y migración rural-urbana en Colombia. Desarrollo y Sociedad, (72), 205-223.

Yang, J. (2013). Social Exclusion and Young Rural-Urban Migrants' Integration into a Host Society in China. The ANNALS of the American Academy of Political and Social Science, 648(1), 52-69.

Zago, N. (2016). Migração rural-urbana, juventude e ensino superior. Revista Brasileira de Educação, 21(64), 61-78. 